\title{
Study to compare the DHFR enzyme activity when exposure to He-Ne laser and UVC radiation in mice
}

\author{
Vyan Faisal Jasim(B.Sc.)*, Falah Naje Nammuk(Ph.D)* \& \\ Shayma`a Jamal Ahmed(Ph.D)** \\ Medical Physics- College of Medicine-Baghdad Un. - Iraq. \\ Anatomy Depart.- College of Medicine-Baghdad Un. - Iraq.
}

\begin{abstract}
In the present work 100 white laboratory mice(Mus musculus) were exposed to He-Ne laser and UVC(1h) radiation for the area of testis. These numbers were divided into five groups : first group is a control (10 mice),second group was exposed to He-Ne laser(27 mice) and this divided into three subgroups for (5min,10min,15min), third group (9 mice) were exposed to UVC for (1h), fourth group (27 mice) were exposed to laser firstly and UVC(1h) with duration time $1 / 2 \mathrm{~h}$ and this group is divided into three subgroups for (5min,10min,15min)for laser radiation, finally fifth group(27 mice) was exposed to UVC (1h)first and laser with duration time $1 / 2 \mathrm{~h}$, which divided to three subgroups for (5min,10min,15min)for laser radiation. In the testis of mice, the results of DHFR enzyme in UVC show the mean value and standard deviation was(318.1583 56.41706$)$, the enzyme activity increased gradually and significantly with UVC(1h)and the correlation between normal and UVC value is significant. The mean value and standard deviation of DHFR enzyme in laser $5 \mathrm{~min}$ was(26.2500 \pm 6.35580$)$, while the mean value and standard deviation of DHFR enzyme in laser 10min was (33.3833 \pm 13.95155$)$. The mean value and standard deviation of DHFR enzyme in laser 15 min

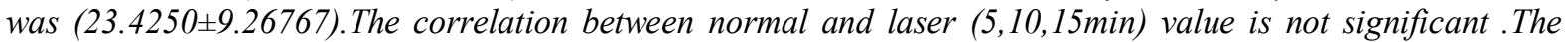

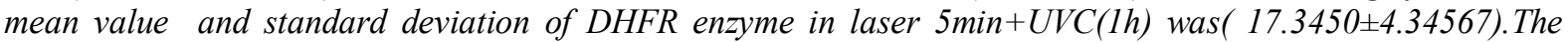
correlation between normal and laser $5 \mathrm{~min}+U V C(1 \mathrm{~h})$ value is significant. The mean value and standard deviation of DHFR enzyme in laser 10min $+U V C(1 \mathrm{~h})$ was (19.4667 18.87891$)$. The mean value and standard deviation of DHFR enzyme in laser15 min $+U V C(1 \mathrm{~h})$ was (39.3400 \pm 31.51039$)$. The correlation between normal and laser10,15min $+U V C(1 \mathrm{~h})$ value is not significant. The mean value and standard deviation of DHFR enzyme in UVC(1h)+laser5min was (55.1833 \pm 22.63580$)$. . The mean value and standard deviation of DHFR enzyme in $U V C(1 h)+$ laser10min was $(42.0383 \pm 11.18429)$.Finally the mean value and standard deviation of

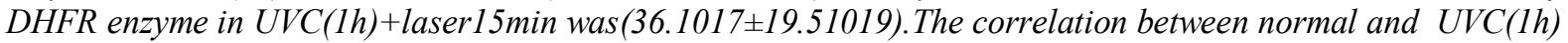
+laser 5,10,15 min value is significant.

key words : He-Ne laser, UVC irradiation ,DHFR.
\end{abstract}

\section{Introduction:}

Laser plays an important role in the medical field for diagnostic and therapeutic applications. Therefore, the selected laser parameters can be used to optimize efficacy while minimizing unwanted side effects and surrounding tissue damage. There were many workers who studied the effect of laser on the skin of different animals (1).

Ultraviolet radiation is an electromagnetic radiation or light having a wavelength greater than $10 \mathrm{~nm}$ but less than $400 \mathrm{~nm}$. Ultraviolet radiation (UV) has a wavelength longer than of $\mathrm{x}$-rays but shorter than that of visible light (2).

UVA :wavelengths between 315 and $400 \mathrm{~nm}$ (3).

UVB : They are Wavelengths in the B range of the ultraviolet (UVB) solar spectrum measured at between 280 and $315 \mathrm{~nm}(4)$.

UVC: It is an Ultraviolet radiation with wavelengths between 200 and $290 \mathrm{~nm}$ (5). This type of radiation is filtered out by the ozone layer so that it doesn't reach the earth's surface (6), and is germicidal which is used in phototherapy (7). The high UVC absorption coefficients of nucleic acids, proteins and aromatic molecules, to an extremely small UVC penetration depth (8). Under certain conditions ultrashort pulses can travel deeper into tissues than $\mathrm{CW}$ radiation, this is because the first part of a powerful pulse may contain enough photons to take all chromophore molecules in the upper tissue layer to excited states, and thus make it more or less transparent for the rest of the pulse. The pulse can literally open a road for itself into tissue $(9,10)$.

\section{Dihydrofolate reductase (DHFR)}

It is a small enzyme that plays a supporting role but an essential role in the building of DNA and other processes (11). It manages the state of folate a snaky organic molecule that shuttles carbon atoms to enzymes that need them in their reactions of particular importance (12), the enzyme thymidylate synthase uses these 
carbon atoms to build thymine bases an essential component of DNA after folate has released its carbon atoms it has to be recycled, this is the job performed by dihydrofolate reductase (13).

DHFR converts dihydrofolate into tetrahydrofolate, a methyl group shuttle required for the de novo synthesis of purines ,thymidylic acid and certain amino acids because of it's small size (18-22) (KD) (14) while the functional dihydrofolate reductase gene has been mapped to chromosome 5 multiple intron processed pseudogenes or dihydrofolate reductase - like genes have been identified on separate chromosomes found in all organisms (15). DHFR has a critical role in regulating the amount of tetrahydrofolate in the cell tetrahydrofolate and it's derivatives are essential for purine and thymidylate synthesis, which are important for cell proliferation and cell growth(16). DHFR plays a central role in the synthesis of nucleic acid precursors, and it has been shown that mutant cells that completely lack DHFR require glycine, an amino acid, and thymidine to grow DHFR has also been demonstrated as an enzyme involved in the salvage of tetrahydrobiopterin from dihydrobiopterin (17).

\section{2-1Animals experiments :}

II. Research Methods:

In order to assess the influence of He-Ne laser plus the UVC irradiation on testis of Mouse the following experiments are performed. 100 adult of albino male mice, weight (25-30) g , are used in this study. Animals were placed in the room ranged temperature (20-25) $\mathrm{C}^{\circ}$ and lighting ranged (12) h light and (12) $\mathrm{h}$ darkness at the "Animal breeding center college of Medical-Baghdad University " and distributed in cages for creditors authority totals and by need experience and for the duration of trials was to give animals water and bush integrated and manufactured by the Center for. Ipa,a for Agricultural research in Abu Ghraib -Baghdad. The mice are divided into five groups and irradiation see table 1.

1. First group: the animals normal, which was control group.

2. Second group: was used to characterize the degree of UVC irradiation on mice testis. The UVC source placed at $20 \mathrm{~cm}$ above the mouse cage, where the final UVC power at the mouse testis surface was $1.2 \mathrm{~mW}$, for $1 \mathrm{~h}$.

3. Third group: was employed to study the influence of He-Ne laser irradiation. A continuous He-Ne laser of wavelength $632.8 \mathrm{~nm}$ with a maximum output power of $1.0 \mathrm{mw}$, and a beam diameter of $3 \mathrm{~mm}$, was employed. The laser beam was directed on testis for a period of $(5,10,15) \mathrm{min}$, which equal to energy dose of $\left(4.2 \mathrm{~J} / \mathrm{cm}^{2}, 8.4 \mathrm{~J} / \mathrm{cm}^{2}, 12.6 \mathrm{~J} / \mathrm{cm}^{2}\right)$.

4. Fourth group: was pre-irradiated by He-Ne laser $\left(4.2 \mathrm{~J} / \mathrm{cm}^{2}, 8.4 \mathrm{~J} / \mathrm{cm}^{2}, 12.6 \mathrm{~J} / \mathrm{cm}^{2}\right)(5,10,15)$ min followed by UVC irradiation, with one hour and wait for $(1 / 2 \mathrm{~h})$ time (for UVC) interval between the two irradiations.

5. Fifth group: was pre-irradiated by UVC light, after one hour and wait for $(1 / 2 \mathrm{~h})$, this group was irradiated by He-Ne $(5,10,15) \mathrm{min}$ laser of $\left(4.2 \mathrm{~J} / \mathrm{cm}^{2}, 8.4 \mathrm{~J} / \mathrm{cm}^{2}, 12.6 \mathrm{~J} / \mathrm{cm}^{2}\right)$, the laser beam was directed on testis.

Table 1 Animals which used in this research

\begin{tabular}{|c|c|c|c|c|}
\hline NO & Types of radiation & $\begin{array}{lr}\text { Number } & \begin{array}{r}\text { of } \\
\text { animals }\end{array} \\
& \text { after } \\
\text { 7days }\end{array}$ & $\begin{array}{r}\text { Number of animals } \\
\text { after } 14 \text { days }\end{array}$ & $\begin{array}{r}\text { Number of animals after } \\
21 \text { days }\end{array}$ \\
\hline $1-$ & Control & 10 & & \\
\hline $2-$ & UVC (1h) & 3 & 3 & 3 \\
\hline $3-$ & $\begin{array}{r}\text { a-Laser(5min) } \\
\text { b-Laser(10min) } \\
\text { c-Laser(15min) }\end{array}$ & $\begin{array}{l}3 \\
3 \\
3 \\
\end{array}$ & $\begin{array}{l}3 \\
3 \\
3 \\
\end{array}$ & $\begin{array}{l}3 \\
3 \\
3 \\
\end{array}$ \\
\hline 4- & $\begin{array}{l}\text { a-Laser(5min)+UVC(1h) } \\
\text { b-Laser(10min)+UVC(1h) } \\
\text { c-Laser(15min)+UVC(1h) }\end{array}$ & $\begin{array}{l}3 \\
3 \\
3 \\
\end{array}$ & $\begin{array}{l}3 \\
3 \\
3 \\
\end{array}$ & $\begin{array}{l}3 \\
3 \\
3 \\
\end{array}$ \\
\hline $5-$ & $\begin{array}{r}\text { a-UVC }(1 \mathrm{~h})+\operatorname{Laser}(5 \mathrm{~min}) \\
\mathrm{b}-\mathrm{UVC}(1 \mathrm{~h})+\operatorname{Laser}(10 \mathrm{~min}) \\
\mathrm{c}-\mathrm{UVC}(1 \mathrm{~h})+\operatorname{Laser}(15 \mathrm{~min})\end{array}$ & $\begin{array}{l}3 \\
3 \\
3\end{array}$ & $\begin{array}{l}3 \\
3 \\
3\end{array}$ & $\begin{array}{l}3 \\
3 \\
3\end{array}$ \\
\hline
\end{tabular}

\section{2-2 The assessment of enzyme activity for DHFR enzyme:}

Mice were killed in separated the spinal cord and explained directly for testis, and put it in test tube , then added (1ml) (PBS). Grind the testis with fine sand by mortar according to (18).

\section{Results:}

In order to check the effect He-Ne laser and UVC on testis of mice and measurement DHFR enzyme activity after 7,14,21 days.

Table 2 show the DHFR enzyme activity after 7,14,21 days after exposing to radiation.

Table 3 show the correlation of DHFR enzyme activity for normal and samples, significant differences in all type of radiation are given. 
Fig 1 show the relation between $\operatorname{UVC}(1 \mathrm{~h}) \&$ laser $(5,10,15)$ min, but Fig 2 observed relation between UVC $(1 \mathrm{~h}) \&$ laser $(5,10,15) \mathrm{min}+\mathrm{UVC}(1 \mathrm{~h})$, also Fig 3 show the relation between UVC $(1 \mathrm{~h}) \& \mathrm{UVC}(1 \mathrm{~h})+$ laser $(5,10,15) \min$,

Finally Fig 4 show the relation between DHFR enzyme activity and all types of radiation.

Table 2-The DHFR enzyme activity after $(7,14,21)$ days after exposing to radiation.

\begin{tabular}{|l|l|}
\hline Radiation & Mean \pm SD \\
\hline 1-Normal & $76.0600 \pm 0.15492$ \\
\hline 2-UVC(1h) & $318.1583 \pm 56.41706$ \\
\hline 3-Laser 5 min & $26.2500 \pm 6.35580$ \\
\hline 4-Laser10min & $33.3833 \pm 13.95155$ \\
\hline 5-Laser15min & $17.4250 \pm 9.26767$ \\
\hline 6-Laser5min+UVC(1h) & $19.4667 \pm 8.87891$ \\
\hline 7-Laser10min+UVC(1h) & $39.3400 \pm 31.51039$ \\
\hline 8-Laser15min+UVC(1h) & $55.1833 \pm 22.63580$ \\
\hline 9-UVC(1h)+laser5min & $42.0383 \pm 11.18429$ \\
\hline 10-UVC(1h)+laser10min & $36.1017 \pm 19.51019$ \\
\hline 11-UVC(1h)+laser15min & \\
\hline
\end{tabular}

Table 3-The correlation of DHFR enzyme activity for normal and samples.

\begin{tabular}{|l|l|l|l|}
\hline Radiation & r-value & Probability & \multicolumn{1}{|c|}{ Relation } \\
\hline 1-Normal\&UVC(1h) & & & significant \\
\hline 2-Normal\&laser 5min & -0.156 & $\geq 0.05$ & No significant \\
\hline 3-Normal\&laser10min & 0.008 & $\geq 0.05$ & No significant \\
\hline 4-Normal\&laser15min & 0.085 & $\geq 0.05$ & No significant \\
\hline 5-Normal\&laser5min+UVC(1h) & -0.123 & $\geq 0.05$ & significant \\
\hline 6-Normal\&laser10min+UVC(1h) & 0.357 & $\geq 0.05$ & No significant \\
\hline 7-Normal\&laser15min+UVC(1h) & 0.067 & $\geq 0.05$ & No significant \\
\hline 8-Normal\&UVC(1h)+laser5min & -0.222 & $\geq 0.05$ & significant \\
\hline 9-Normal\&UVC(1h)+laser10min & -0.117 & $\geq 0.05$ & significant \\
\hline 10-Normal\&UVC(1h)+laser15min & -0.0003 & $\geq 0.05$ & significant \\
\hline
\end{tabular}

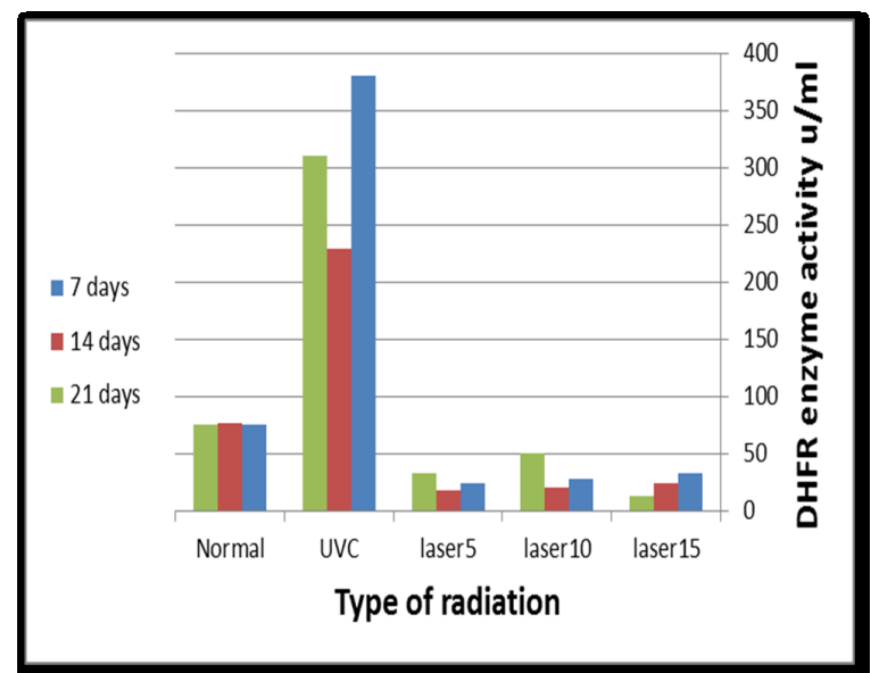

Fig .1. Relation between UVC(1h)\&laser(5,10,15)min. 


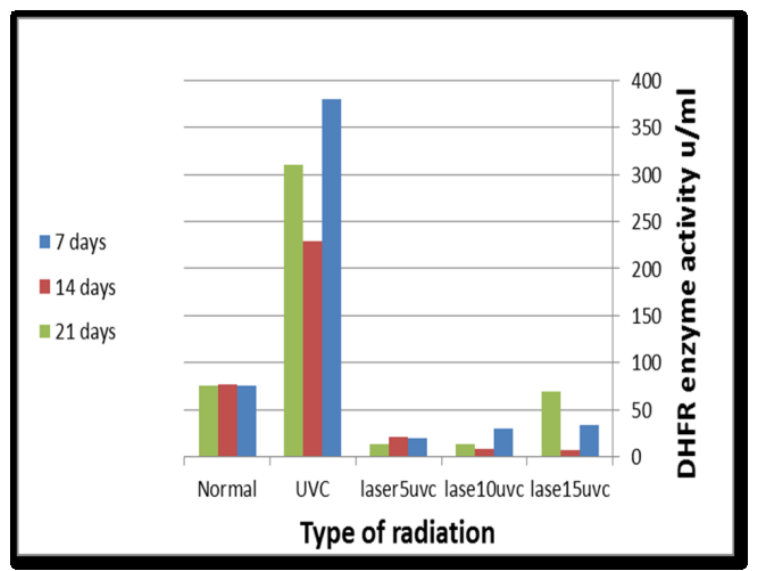

Fig .2. Relation between UVC(1h)\& laser(5,10,15)min +UVC(1h).

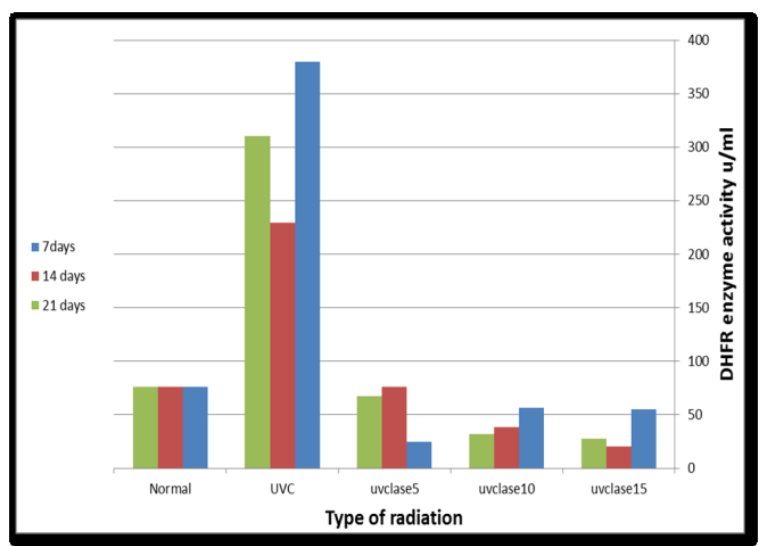

Fig .3.Relation between UVC(1h)\&UVC(1h)+laser(5,10,15)min.

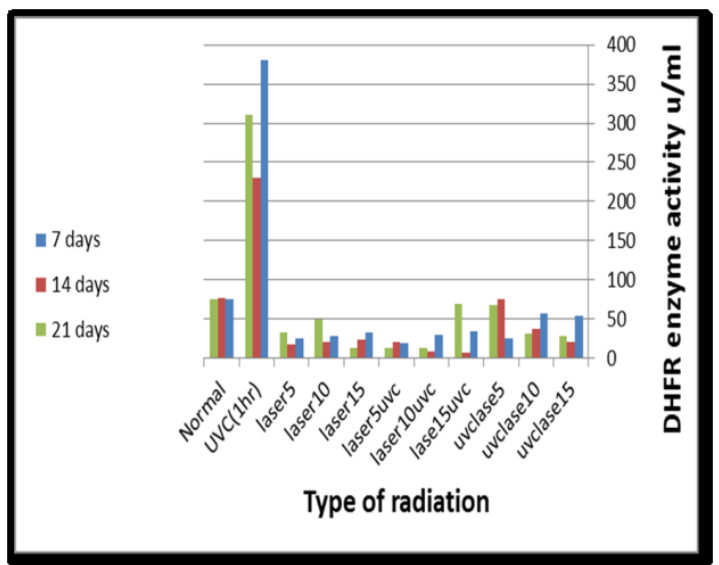

Fig .4.Relation between DHFR enzyme activity and all types of radiation.

\section{Discussion :}

In the UVC(1h) after7 days the value of the DHFR enzyme (379.8) was highly compare with normal (75.96), but after 14 days DHFR value was (229.6) highly compare with normal (76.2) too. After 21 days DHFR value was (310.05) highly compare with normal(75.9), high value of DHFR with UVC(1h) after 7 days, less value after 14 days, value of DHFR after 21 days less from after 7 days but upper from 14 days, the effect of UVC(1h) was highly, which increased DHFR enzyme as shown in (Fig .1), in other hand the correlation between normal and UVC (-0.156) was significant as shown in table 3 .

In the laser 5 min after 7 days DHFR value was (24.73) low compare with normal (75.96), but after 14 days DHFR value was (17.66) low compare with normal (76.2), after 21 days DHFR value was (32.86) low compare with normal (75.9) as shown in (Fig .1), also the correlation between normal and laser $5 \mathrm{~min}(0.195)$ was no significant as shown in table 3. 
In the laser10 min after 7 days DHFR value was (28.6) low compare with normal (75.96), but after 14 days DHFR value (20.2) was low compare with normal (76.2), after 21 days DHFR value (50.1) was low compare with normal (75.9) as shown in (Fig . 1), the correlation between normal and laser 10 min (0.008) was no significant as shown in table 3 .

In the laser 15 min after 7 days DHFR value (33.5) was low compare with normal (75.96), but after 14 days DHFR value (23.85) was low compare with normal (76.2), after 21 days DHFR value (12.9) was low compare with normal (75.9) as shown in (Fig .1), the correlation between normal and laser 15 min (0.085) was no significant as shown in table 3 .

So in the laser $(5,10,15)$ min the high value of DHFR in laser 10min (50.1) after 21 days, the less value of DHFR after 21 days in laser 15 min (12.9).

In the laser 5min+UVC(1h) after 7 days DHFR value was (19.43)low compare with normal (75.96) but after 14 days DHFR value (21.2) was low compare with normal (76.2), after 21 days DHFR value (13.2) was low compare with normal (75.9) as shown in (Fig .2) , the correlation between normal and laser5min+UVC $(1 \mathrm{~h})(-0.123)$ was significant as shown in table 3.

In the laser 10min+UVC(1h), after 7 days DHFR value (30.3)was low compare with normal (75.96), but after 14 days DHFR value (8.83) was low compare with normal (76.2), after 21 days DHFR value (13.25) was low compare with normal (75.9) as shown in (Fig.2), the correlation between normal and laser10min+UVC(1h) (0.357) was no significant as shown in table 3 .

In the laser15min+UVC(1h) after 7 days DHFR value(33.74)was low compare with normal (75.96) but after 14 days DHFR value(7.4)was low compare with normal (76.2),after 21 days DHFR value(69.7)was low compare with normal (75.9) as shown in (Fig .2), the correlation between normal and laser15min+UVC $(1 \mathrm{~h})(0.067)$ was no significant as shown in table 3.

So in the laser(5,10,15)min+ UVC(1h)after 7,14,21 days high value in laser15min+UVC(1h)(69.7) after 21 days , less value in laser $15 \mathrm{~min}+\mathrm{UVC}(1 \mathrm{~h})(7.4)$ after 14 days.

In the UVC(1h)+laser5min after 7 days DHFR value(24.73)was low compare with normal (75.96), after 14 days DHFR value(75.9)was low compare with normal (76.2), after 21 days DHFR value(67.57)was low compare with normal (75.9) as shown in (Fig .3), the correlation between normal and UVC(1h) +laser5min(0.222 ) was significant as shown in table 3.

In the UVC(1h)+laser10min after 7 days DHFR value( 56.53)was low compare with normal (75.96) ,but after 14 days DHFR value(38.2)was low compare with normal (76.2),after 21 days DHFR value(31.8) was low compare with normal (75.9) as shown in (Fig . 3), the correlation between normal and UVC(1h) + laser10min(-0.117) was significant as shown in table 3 .

In the UVC(1h)+laser15min after 7 days DHFR value( 54.76) was low compare with normal (75.96) ,but after 14 days DHFR value(20.3) was low compare with normal (76.2), after 21 days DHFR value(27.8) was low compare with normal (75.9) as shown in (Fig .3), the correlation between normal and UVC(1h) + laser $15 \min (-0.0003)$ was significant as shown in table 3 .

So in the UVC $(1 \mathrm{~h})+$ laser $(5,10,15) \mathrm{min}$ the high value after 14 days $(75.9)$ in the $\mathrm{UVC}(1 \mathrm{~h})+$ laser $5 \mathrm{~min}$ but the less value after 14 days in the UVC (1h) +laser $15 \mathrm{~min}(20.3)$.

The high value of DHFR enzyme in all types of radiation after 7,14,21 days in UVC(1h) after 7 days value (379.8) and less value of DHFR enzyme after 7,14,21 days in laser $15 \mathrm{~min}+\mathrm{UVC}(1 \mathrm{~h})$ (7.4) after 14 days as shown in (Fig .4).

Through this study the results show the effect UVC(1h) height but laser could to make protection against UVC by decreased DHFR enzyme.

These results indicate that UVC (1h) increase enzyme level and He-Ne laser decrease enzyme level and this in agreement with (19).

The enzyme activity increased gradually and significantly with UVC(1h) and this in agreement with (20), the activity increased gradually, the rate of increase was relative to the increase concentration of the drag.

The inhibit of the enzyme dihydrofolate reductase competing with it's natural substrates, and so block the production of tetrahydrofolate, an essential cofactor for DNA synthesis(21).

As conclusion: that if He-Ne laser is used with UVC then it will give protection against UVC. UVC (1h) increase enzyme level and He-Ne laser decrease enzyme level.

\section{References:}

[1]. Abuarra A.,Abuarra B.,Abur B.S.,Singh G.K.C.,AlSadi Z.,MahmoodTg.L.R., Omar K. and MatJafr M.Z., 2012,The effects of different laser doses on skin,International Journal of the Physical Sciences , 7(3): $400-407$,ISSN 1992 - 1950,DOI: 10.5897/IJPS11.1718

[2]. Helmenstine A.M.,2013,Ultraviolet Radiation Definition,Chemistry Glossary Definition of Ultraviolet Radiation, About .com Chemistry.

[3]. Svobodova A., Walterova D. and Vostalova J., 2006,Ultraviolet Light Induced Alteration to the Skin, Biomed Pap Med Fac Univ Palacky Olomouc Czech Repub,150(1):25-38. 
[4]. Li J., Zhang Z., Liu F., Liu Q., Gan W., Chen G., Lim M.L.M. and Li D.,2008, UVB-Based Mate-Choice Cues Used by Females of the Jumping Spider Phintella vittata, Current Biology,18(9):699-703, DOI 10.1016/j.cub.2008.04.020.

[5]. Thai T.P., Keast D.H., Campbell K.E., Woodbury M.G. and Houghton P.E.,2005, Effect of ultraviolet light C on bacterial colonization in chronic wounds, Ostomy Wound Manage, 51(10):32-45.

[6]. Young C.,2009, Solar ultraviolet radiation and skin cancer, Oxford Journals,59:82-88, ISSUE (2), doi: 10.1093/occmed/kqn170.

[7]. Tasi M.H.,Aki R.,Amoh Y.,Hoffman R.M.,Katsuoka K.,Kimura H.,Lee C .and Chang Ch.,2010,GFP-Fluorescence-guided UVC Irradiation Inhibits Melanoma Growth and Angiogenesis in Nude Mice, Anticancer Research,30:3291-3294.

[8]. Peng Q.,JuzenieneA.,Chen J.,Svaas and L.O. ,WarloeT. ,Giercksky K.E. and Moan J.,2008,Lasers in medicine,Reportson Progressin Physics ,71:28, doi: 10.1088/0034-4885/71/5/ 056701.

[9]. Pogue B .W., Lilge L., Patterson M .S., Wilson B. C. and Hasan T.,1997,Absorbed photodynamic dose from pulsed versuscontinuous wave light examined with tissue-simulatingdosimeters Appl. Opt. 367257.

[10]. Sterenborg H. J. and van Gemert M. J ., 1996,Photodynamic therapy with pulsed light sources: a theoretical analysisPhys, Med. Biol. 41835 .

[11]. Adinarayana K.P.S .and Devi R.K.,2011,Protein-Ligand interaction studies on 2, 4, 6- trisubstituted triazine derivatives as antimalarial DHFR agents using AutoDock,USA National Library of MedicineNational Institutes of Health(PMC),national center for biotechnology information ,Bioinformation, 6(2):74-77.

[12]. Prakash N.,Patel S.,Faldu N.J.,Ranjan R. and Sudhee D., 2010,Molecular Docking Studies of Antimalarial Drugs for Malaria,Journal of Computer Science \& Systems Biology, 3(3): 070-073, Issue 3, doi:10.4172/jcsb.1000059.

[13]. Goodsell D.,2002,DihydrofolateReductase,RCSB Protein Data Bank,EM Data Bank,An Educational Resource for Exploring a Structural View of Biology.

[14]. Shakya S.,Kasturi K. and Rao K.R.S.S.,2010, Dihydrofolate reductasea Target for antimalarial DRUG ,An International Journal of Advances In Pharmaceutical Sciences, 1(1).

[15]. Nano R ., 2000,Dihydrofolate reductase activity in the erythroblasts of patients with 59-syndrom, Haematologica ,85(7):765-784.

[16]. Schnell J.R., Dyson H .J.and Wright P.E., 2004,"Structure, dynamics, and catalytic function of dihydrofolate reductase.", Annual Review of Biophysics and Biomolecular Structure ,33 (1): 119-40, doi:10.1146/annurev.biophys.33.110502.133613. PMID 15139807.

[17]. Urlaub G. and Chasin L.A., 1980,"Isolation of Chinese hamster cell mutants deficient in dihydrofolate reductase activity", Proc. Natl. Acad. Sci. U.S.A, 77 (7): 4216-20, doi:10.1073/pnas.77.7.4216. PMC 349802.PMID 6933469.

[18]. Morton R.K.,1954,The purification of alkaline phosphatase of animal tissue,Biochem.J,27:595-603.

[19]. Chen Yi.P.,2009,Response of antioxidant defense system to laser radiation apical meristem of Isatis indigotica seedlings exposed to UV-B,Plant Signaling Behavior ,4(7):571-573.

[20]. Jaffer Z.M.T., Ammash H.S. and Shubber E.K.,2001, Resistant Chinese Hamster Lung Fibroblast Cells ,AgrBiol Res Center,44(1,2):36-43.

[21]. Gready J.E.,1979,Dihydrofolate reductase: the current story,Nuffield Foundation Fellow in the Physical Chemistry Laboratory, Oxford, Macmillan Journals,282:674-675, issue 5740. 